Ann. Biol. anim. Biol. Biophys., I97I, 11 (3), 495-498.

\title{
TECHNIQUE D'EXAMEN CELIOSCOPIQUE DES OVAIRES DE LA TRUIE
}

\author{
A. LOCATELLI \\ Station de Physiologie de la Reproduction, \\ Centre de Recherches de Tours, I. N. R. A., \\ 37 - Nouzilly
}

Chez la Truie, l'examen des ovaires in vivo est réalisé en général par laparotomie. Une méthođe d'examen après mise en place de canules péritonéales a été décrite par BETTERIDGr et RAESIDE (1962).

La laparoscopie, technique relativement ancienne chez l'Homme (OTT, Igor ; KELLING, I902) et qui a bénéficié de multiples modifications (Melvin et CoHen, I968) a été utilisée récemment chez la Brebis et la Vache pour l'examen des ovaires (Thimonier et MaulEon, I967-I 969; Megale et al., 1956 ; Roberts, 1968; Mariana, 1969). Nous indiquerons ici les possibilités d'utiliser chez la truie une techniques de cœlioscopie comparable.

\section{MATÉRIEL, E'T MÉTHODES}

$$
\text { A. - Matériel }
$$

L'appareil utilisé est un endoscope "Storz » de diamètre I I mm.

Une tige de manipulation complète l'appareillage. à $\mathbf{r} 70 \mathrm{~kg}$.

Les observations ont été réalisées sur 80 truies nullipares de race Large White pesant 120

$$
\text { B. - Technique d'observation }
$$

L'animal anesthésié est installé en décubitus dorsal sur une table inclinée à $30^{\circ}$ environ. Après pose de champs stériles, deux incisions de $I, 5 \mathrm{~cm}$ sont faites au bistouri à $5 \mathrm{~cm}$ en arrière de l'ombilic et à $3 \mathrm{~cm}$ de part et d'autre de la ligne médiane. Un trocard est enfoncé verticalement dans l'une des incisions. Le laparoscope vient remplacer le mandrin et un pneumopéritoine est créé.

Un second trocard est alors installé dans la deuxième incision. Il est destiné à la mise en place d'une tige de manipulation servant à écarter l'utérus et le pavillon.

Après examen, des antibiotiques liquides sont introduits par le fourreau du trocard et l'incision est suturée par une agrafe. 


\section{EFFICACITÉ DE LA MÉTHODE-RÉSULTATS}

\section{A. - Aspect qualitatif}

La visibilité est bonne pour l'appréciation des formes, des couleurs ou du relief.

Cela permet de distinguer sans risque d'erreur sur l'ovaire les follicules, les corps jaunes fonctionnels récemment formés, les corps jaunes florides et les corps jaunes en cours de régression. On peut également estimer les dimensions par comparaison avec le diamètre des organes voisins, en particulier celui de la trompe.

\section{B. - Aspect quantitatif}

Deux séries de mesures permettent d'estimer l'efficacité de la méthode cœlioscopique pour le dénombrement des follicules ou des corps jaunes.

Le premier examen est réalisé pendant l'œestrus et l'ensemble des follicules mûrs et des corps jaunes en régression sont comptés. Le second examen est effectué quelques jours plus tard et les valeurs obtenues sont comparées.

a) Sur 30 truies, l'endoscopie réalisée durant l'œstrus a été suivie d'un examen direct des ovaires par laparotomie ou abattage réalisé 5 à 6 jours après la première intervention. Les résultats sont indiqués dans le tableau I : 70 p. Ioo des 30 dénombrements sont exacts à une unité près, soit par excès, soit par ciéfaut. Dans un cas seulement, l'erreur est de plus de 2 formations.

TABLEAU I

Précision des examens calioscopiques

\begin{tabular}{|c|c|c|c|c|c|}
\hline \multicolumn{3}{|c|}{$\begin{array}{l}\text { Répartition des examens suivant } \\
\text { le niveau de précision obtenu }\end{array}$} & \multicolumn{3}{|c|}{ Nombre de formations comptées (1) } \\
\hline $\begin{array}{c}\text { Différence entre } \\
\text { endoscopie et } \\
\text { vérification }(2)\end{array}$ & Nombre de cas & 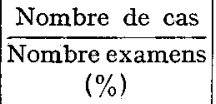 & Endoscopie & Vérification & $\begin{array}{l}\text { Différence totale } \\
\text { entre endoscopie } \\
\text { et vérification }\end{array}$ \\
\hline 0 & 8 & 26,6 & 100 & 100 & 0 \\
\hline 1 & 13 & 43,3 & $\begin{array}{l}63 \\
88\end{array}$ & $\begin{array}{l}58 \\
96\end{array}$ & $13 \begin{cases}5 & \text { par excès } \\
8 & \text { par défaut }\end{cases}$ \\
\hline 2 & 8 & 26,6 & $\begin{array}{l}37 \\
53\end{array}$ & $\begin{array}{l}31 \\
63\end{array}$ & $16\left\{\begin{aligned} 6 & \text { par excès } \\
10 & \text { par défaut }\end{aligned}\right.$ \\
\hline 3 et plus & 1 & 3,3 & 11 & 14 & 3 par défaut \\
\hline Totaux & 30 & 100 & 352 & 362 & 32 \\
\hline
\end{tabular}
l'ovaire.

(1) Nombre de formations comptées = somme des follicules mûrs et des corps jaunes observés sur

(2) Différence entre le nombre de formations comptées lors de l'endoscopie et de la vérification pour une même truie :

0 - groupe des truies où il n'y a pas d'erreur.

1 = groupe des truies où il y a une erreur de 1 corps jaune. 
La quantité totale des corps jaunes et follicules dénombrés par examen cœlioscopique des ovaires des 30 truies dejl'ensemble du lot diffère de ro (par défaut) du nombre de corps jaunes effectivement présents, soit 0,33 corps jaunes par truie.

TABLEAU 2

Répétabilité entre deux endoscopies

\begin{tabular}{|c|c|c|c|c|c|}
\hline \multicolumn{3}{|c|}{$\begin{array}{l}\text { Répartition des examens suivant } \\
\text { le niveau de précision obtenu }\end{array}$} & \multicolumn{3}{|c|}{ Nombre de formations comptées $\left({ }^{\mathbf{1}}\right)$} \\
\hline $\begin{array}{c}\text { Différence } \\
\text { entre deux } \\
\text { endoscopies }\left({ }^{2}\right)\end{array}$ & Nombre de cas & 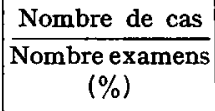 & Minimum & Maximum & $\begin{array}{l}\text { Différence totale } \\
\text { entre les deux } \\
\text { endoscopies }\end{array}$ \\
\hline 0 & 17 & 38,6 & 199 & 199 & 0 \\
\hline 1 & 18 & 40,9 & 243 & 261 & $18\left\{\begin{aligned} 6 & \text { par excès } \\
12 & \text { par defaut }\end{aligned}\right.$ \\
\hline 2 & 6 & 13,6 & 78 & 90 & $12\left\{\begin{array}{l}6 \text { par excès } \\
6 \text { par défaut }\end{array}\right.$ \\
\hline 3 et plus & 3 & 6,8 & 26 & 37 & 11 par défaut \\
\hline Totaux & 44 & 100 & 546 & 587 & 41 \\
\hline
\end{tabular}
l'ovaire.

(1) Nombre de formations comptées = somme des follicules mûrs et des corps jaunes observés sur

(2) Différence entre le nombre de formations comptées lors des deux endoscopies pour une même truie :

$0=$ groupe des truies où il n'y a pas eu d'erreur.

$1=$ groupe des truies où il $\mathrm{y}$ a eu une erreur de 1 corps jaune.

b) Sur 44 truies, deux examens cœlioscopiques ont eu lieu à 96 heures d'intervalle (tabl. 2). La concordance entre les deux examens, à une unité près, est observée dans $79,5 \mathrm{p}$. Ioo des cas.

La précision du diagnostic est bonne, mais celle du dénombrement varie avec la quantité de corps jaunes, comme chez la Vache (MarIaNa, I969) et avec les différentes périodes du cycle œstrien : la concordance à une unité près est observée dans 42,6 p. Ioo des cas seulement pour le comptage des corps jaunes récemment formés, qui sont très difficiles à identifier sur l'ovaire, contre $83,7 \mathrm{p}$. roo des cas pour l'examen de follicules à la veille de l'ovulation. L'habitude de l'observateur et le perfectionnement du matériel doivent normalement augmenter la précision du dénombrement.

Les inconvénients expérimentaux entraînés par l'anesthésie générale ou la manipulation des organes sont les mêmes que pour une laparotomie ; certains risques en sont supprimés. 


\section{SUMMARY}

\section{COELIOSCOPIC VISUAL,IZATION OF SOW OVARIES}

A cœlioscopic technique for visualizing sow ovaries enabled us to observe and differenciate follicles and new, fully developed or regressing corpora lutea.

The accuracy of the technique was satisfactory :

- 7o p. Ioo of the countings were accurate within I unit, with 30 sows cœlioscopically observed when in cestrous and directly examined (laparotomy or slaughter) 5 to 6 days later as a control (table I).

- $79 \mathrm{p}$. Ioo of the countings were in accordance within I unit with 44 sows cœlioscopically observed twice at $96 \mathrm{hr}$. interval (table 2).

\section{RÉFÉRENCES BIBLIOGRAPHIQUES}

BetTeridge J. K., RAESIDE J. I., 1962, Observation of the ovary by peritoneal cannulation in pigs. Res. Vet. Sci., 3, 390-398.

Du Mesnil du Buisson F., Mauléon P., Locatelli A., Mariana J.-C., I97o. Modification du moment et de l'étalement des ovulations après maîtrise du cycle sexuel de la truie. L'inhibition de l'ovulation, Masson éd., I vol., 225-234.

Kelling G., 1902. Ủber Oesophagoskopie, Gastroskopie und Cölioskopie. Med. Wschr., München, 49, $2 x$.

Mariana J.-C., I969. Technique d'examen in wivo des ovaires chez la vache. Ann. Biol. anim. Bioch. Biophys., 9, 657-659.

Megale G., Fincher M. G., McEntee K., I956. Peritoneoscopy in the cow visualization of the ovaries, oviducts and uterine horns. Cornell Vet., 156, Iog-I2X.

MrLvin R., Cohen M. D., I968. Culdoscopy vs. Periotoneosocpy. Obst. Gynec., 31, 3, 3ro-32i.

OTT D. O., Igor. Veritroscopic illumination of the abdominal cavity in pregnancy. Zakus $i$ Zhensk Bolez, 15, 7-8.

RoBerts E. M., 1968. Cyclic changes in the ovaries of anoestrous ewes. VI Congr. intern. reprod. anim. insém. artif., Paris, vol. $x, 723-726$.

Thimonier J., MaulÉon P., I967. Variations saisonnières des activités hypophysaires des brebis de race Ile-de-France. Coll. Int. Photorégulation de la reproduction chez les oiseaux et les mammifères, Montpellier, $17-22$ juillet 1967 .

Thimonier J., MaUlÉon P., I969. Variations saisonnières du comportement d'œstrus et des activités ovarienne et hypophysaire chez les ovins. Ann. Biol. anim. Bioch. Biophys., 9, 233-250. 